\title{
Congenital superior vena cava (SVC) stenosis and obstructed supracardiac total anomalous pulmonary venous connection (TAPVC) - A surgical perspective
}

\author{
Sachin Mahajan ${ }^{1}$, Sudhansoo Khanna ${ }^{1}$, Manojkumar Rohit ${ }^{1}$, Nirupam Chakraborty ${ }^{1}$, and \\ Ankit Singhal ${ }^{1}$ \\ ${ }^{1}$ Post Graduate Institute of Medical Education and Research
}

April 28, 2020

\begin{abstract}
Congenital superior vena cava (SVC) stenosis is a very rare anomaly especially in pediatric population. Co-existence with obstructed supracardiac total anomalous pulmonary venous connection (TAPVC) has never been reported. Clinical examination should prompt detailed and focused evaluation for this treatable etiology. SVC stenosis, although causing SVC syndrome, may decrease the severity of pulmonary venous hypertension by limiting the amount of blood in obstructed common chamber. Pericardial patch augmentation can cure SVC stenosis, and may allow for growth potential as well. We describe a case of congenital SVC stenosis in a case of obstructed supra-cardiac TAPVC in a 3 month old infant, managed successfully.
\end{abstract}

Text

Introduction

Superior vena cava stenosis is an uncommon anomaly, usually seen as postoperative complication following cardiac surgery. ${ }^{1,2}$ True congenital SVC stenosis is even rarer and can be lethal, if not addressed in time. Rapid resolution of symptoms is possible by stent angioplasty. ${ }^{3}$ Association with obstructed TAPVC can lead to pulmonary venous hypertension, along with SVC syndrome. Physical findings should prompt detailed and focused evaluation for timely intervention. To the best of our knowledge, congenital SVC stenosis in association with obstructed supracardiac total anomalous pulmonary venous connection (TAPVC) has never been reported

\section{Case report}

Informed written consent was taken from the patient's guardian for the publication. A 3 month old infant weighing $2.2 \mathrm{Kg}$ was hospitalized with respiratory distress. On examination, patient was tachypneic, cyanosed and dilated veins were visualized all over the chest and abdomen. A faint ejection systolic murmur was heard at left upper sternal border and per abdomen revealed mild hepatomegaly. Chest X-ray revealed slight cardiomegaly with prominent hilar markings. Electrocardiogram (ECG) was consistent with features of right ventricular hypertrophy (RVH). Mild hepatomegaly was observed on ultrasound abdomen. Transthoracic echocardiography (TTE) revealed anomalous pulmonary venous confluence behind left atrium (LA), draining directly into superior vena cava (SVC) from right lateral aspect at junction of SVC with right atrium (RA). There was a discrete narrowing at this confluence. SVC was grossly dilated proximal to the stenotic site with flow velocity of $2.5 \mathrm{~m} / \mathrm{sec}$ across the narrowed segment. RA and right ventricle (RV) were dilated and an ostium secondum atrial septal defect (OS-ASD) was also present. Contrast enhanced computed tomography (CECT) chest, revealed that all 4 pulmonary veins (PV) joined to form a common chamber 
(CC) behind LA which was draining into SVC at SVC-RA junction with discrete narrowing at the junction. There was bulbous dilatation of SVC proximal to stenotic site (Figure 1).

With provisional diagnosis of obstructed supracardiac total anomalous pulmonary venous connection (TAPVC) and congenital SVC stenosis, patient was taken up for surgery. Per-operatively, proximal SVC as well bilateral innominate veins were hugely dilated. Circumferencial stenosis at lower end of SVC (approximately 1 $\mathrm{cm}$ in length), starting at SVC-CC junction and extending distally till its termination into RA was observed (Figure 2). Drainage of the CC was into the lower end of SVC at its right lateral aspect by an opening that was roughly 3-4 $\mathrm{mm}$ in size. RA, RV were dilated. Cardiopulmonary bypass (CPB) was established with aortobicaval cannulation, with special emphasis on high SVC cannulation. After cardioplegic arrest, the heart was rotated anticlockwise around its axis and dropped into right plural cavity. Anastomosis of LA with CC was done. SVC was widely incised on its lateral aspect. Circumferential fibrosis was observed within the distal part of SVC emanating from the drainage site of CC into SVC (Figure 3). The connecting vein between CC and SVC was doubly ligated from outside, and SVC was augmented with a rectangular pericardial patch (Figure 4). Patient was weaned off CPB with inotropic support and nitric oxide (NO). Trans-esophageal echocardiography (TEE) intra-operatively, ruled out any significant gradient across SVCRA junction, and CC-LA anastomosis. Delayed sternal closure was done and patient had an uneventful recovery.

\section{Discussion}

SVC stenosis in pediatric population is usually because of postoperative sequelae to prior cardiac surgery. ${ }^{1}$ "True" Congenital SVC stenosis is an extremely rare entity. To the best of our knowledge, co-existence of this anomaly with obstructed TAPVC, has never been reported. We believe the etiology in our case was transmural fibrosis emanating from the draining ostium of common chamber, involving SVC circumferentially.

Symptomatic SVC obstruction may increase venous pressure, depicted as SVC syndrome, but the degree and severity of symptoms depend on the extent and rate of progression of narrowing. ${ }^{2}$ In our case disproportionate edema in the SVC distribution was absent, indicating gradual development of narrowing in-utero. Also massive dilation of proximal SVC, could have acted as a reservoir. In the era when a TTE is performed as a standalone test, subtle findings can be overlooked. ${ }^{4}$ Disproportionate edema or dilated veins in the SVC distribution are critical physical findings that should prompt focused evaluation for this etiology. Focused TTE and CT can readily establish the diagnosis in these sick infants.

Neonates and infants with obstructed TAPVC can present with severe cyanosis, pulmonary hypertension, and low cardiac output, requiring emergency surgical intervention. ${ }^{5}$ In our patient, the drainage of CC was almost atretic, but the lungs were not congested. Coexisting critical SVC stenosis can limit the amount of blood going towards the common chamber. We are not sure, whether coexisting SVC stenosis can decrease the severity of pulmonary venous hypertension, by limiting the amount of blood flow into the obstructed common chamber.

Management of SVC obstruction needs to be tailored according to the etiology and coexisting malformations. For isolated SVC stenosis, stenting is becoming the modality of choice compared to surgery due to its reduced post-procedure morbidity and faster recovery time. ${ }^{2}$ Intravascular stent placement is safe and effective even in term infants. ${ }^{3}$ However lack of growth potential and recurrence of sympyoms, mandates reinterventions. Considering the small vessel size as well the growth potential in pediatric population, surgical repair with patch augmentation is ideal, especially in the setting of an associated CHD which needs surgical correction. In our patient, associated obstructed supracardiac TAPVC mandated surgical intervention for SVC stenosis. We resorted to pericardial patch augmentation of SVC along with anastomosis of common chamber to the posterior wall of LA.

The hemodynamic effect produced by a combination of systemic venous hypertension and pulmonary venous hypertension can be extremely lethal especially in infants. Clinical condition should prompt focused evaluation by TTE and CECT. We believe surgical repair with a pericardial patch, can be curative for SVC stenosis in pediatric population, by providing growth potential. 


\section{Author contributions}

Dr. Sachin Mahajan - Conception and design.

Dr. Sudhansoo Khanna - Data analysis, Drafting article

Dr. Manojkumar Rohit - Critical revision of article.

Dr. Nirupam Sekhar Chakraborty - Data collection.

Dr Ankit Singhal- Data collection.

\section{References}

1. Jayakumar A, Hsu DT, Hellenbrand WE, Pass RH. Endovascular stent placement for venous obstruction after cardiac transplantation in children and young adults. Catheter Cardiovasc Interv 2002;56:383-386.

2. Yubbu P, Jin OY, Itam MA, Leman H, Tiong KG. Two Case Reports of Emergency Stenting of Superior Vena Cava Obstruction Following Cardiac Surgery. Int J Clin Cardiol 2019;6:139. doi. org/10.23937/2378-2951/1410139.

3. Ro PS, Hill SL, Cheatham JP. Congenital superior vena cava obstruction causing anasarca and respiratory failure in a newborn: Successful transcatheter therapy. Cathet Cardiovasc Intervent 2005;65:60-65. doi:10.1002/ccd.20356.

4. Behrle N, Divekar A. Congenital superior vena cava syndrome: a rare cause of nonimmune hydrops fetalis. J Am Coll Cardiol 2018; 71(11): A2485. doi: 10.1016/S0735-1097(18)33026-2.

5. Lo-A-Njoe SM, Blom NA, Bökenkamp R, Ottenkamp J. Stenting of the vertical vein in obstructed total anomalous pulmonary venous return as rescue procedure in a neonate. Catheter Cardiovasc Interv 2006;67(5):668-670. doi:10.1002/ccd.20715.

\section{Figure legends}

Figure 1 CECT- Thorax showing bulbous dilatation of SVC proximal to stenotic site.

Figure 2 Intra-operative photograph showing dilated SVC proximal to stenosis, and CC draining into lateral aspect of SVC-RA junction via a connecting vein (Note- Yellow vessel loop around the connecting vein).

Figure 3 Intra-operative photograph after laying opening of SVC on its lateral aspect showing circumferential fibrosis within the distal part of SVC emanating from the drainage site of CC into SVC.

Figure 4 Intra-operative photograph showing pericardial patch augmentation of SVC. 

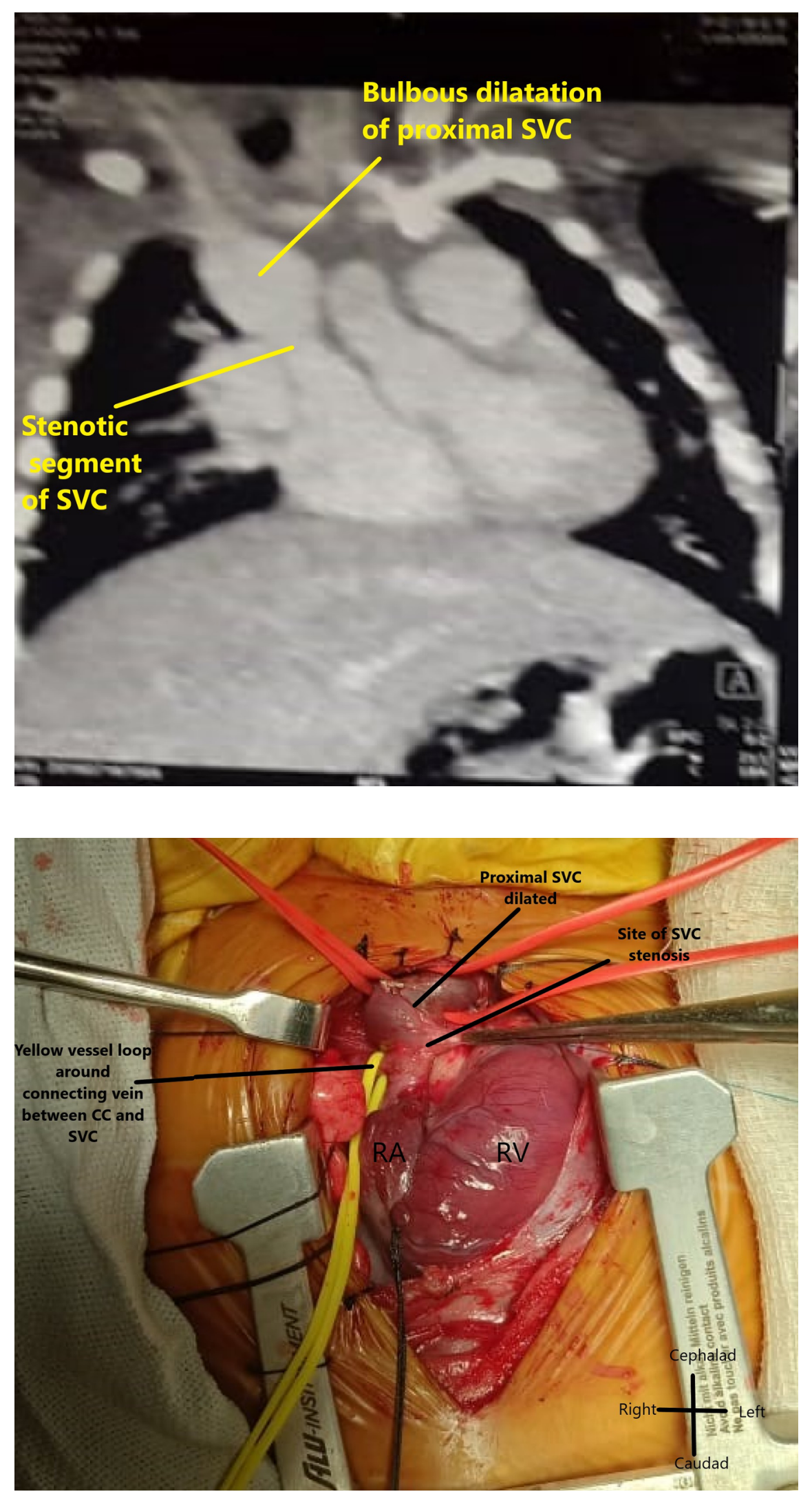

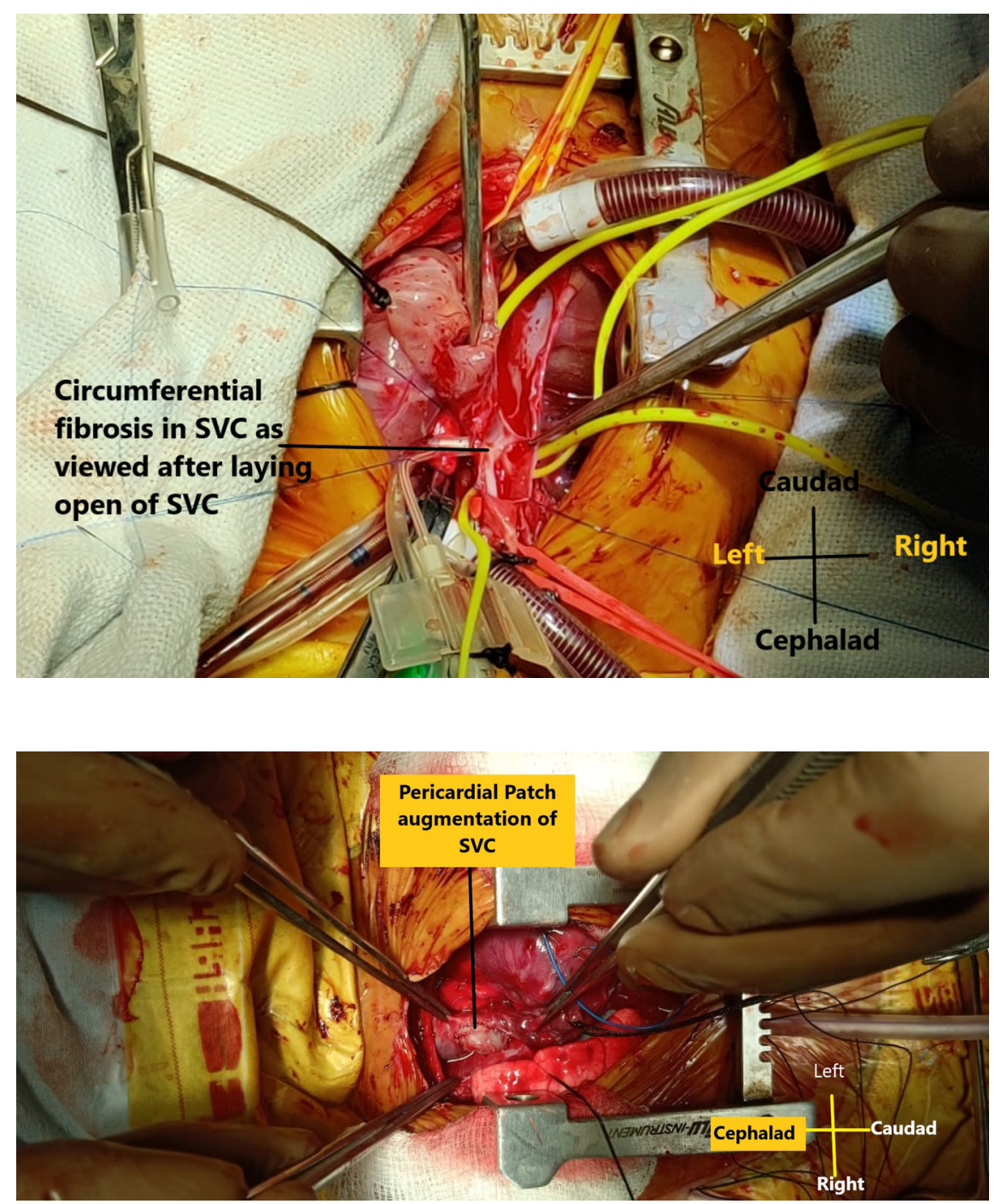